\title{
小腔游仆虫形态学、个体发育与分子系统学研究
}

\author{
张 雪 ${ }^{1}$ 王芯 ${ }^{1}$ 樊阳波 ${ }^{1,2}$ 罗晓甜 ${ }^{1}$ 胡晓钟 ${ }^{1}$ 高 凤 ${ }^{1 *}$ \\ 1 (中国海洋大学水产学院, 山东青岛 266003) \\ 2 (哈尔滨工业大学(深圳)土木与环境工程学院, 广东深圳 518055)
}

\begin{abstract}
摘要: 游仆类是纤毛虫中进化最为复杂和高等的一大类群, 为了进一步探索和完善游仆类的多样性, 本研究利用 活体观察、蛋白银和银浸法染色技术对采自青岛小西湖的小腔游仆虫(Euplotes aediculatus)的形态学及细胞发生学 进行了详尽的研究, 并在完整的形态学及发生学研究基础上, 测定了小腔游仆虫的核糖体小亚基基因(SSU rDNA) 序列, 通过序列比较和分子系统树构建等方法, 对小腔游仆虫的系统地位进行了分析。结果表明: 本种鉴别特征为 9 根额腹棘毛, 5 根横棘毛, 2 根缘棘毛, 2 根尾棘毛, 8 列背触毛, double-eurystomus型银线系。发生学特征包括: (1)后仔 虫口原基在表皮下独立发生, 前仔虫完全继承老口围带; (2)额-腹-横棘毛原基从左向右按照 3:3:3:2:2的模式形成 额腹棘毛和横棘毛; (3)前后仔虫最左侧额腹棘毛分别由独立产生的原基形成; (4)缘棘毛原基独立发生; (5)初级背 触毛原基来自虫体中部老结构的反分化; (6)前后仔虫尾棘毛分别来自最右侧2列背触毛原基和老背触毛列末端; 这些特征显示出游仆虫属个体发生模式的高度保守性。分子系统分析与形态学数据一致, 即游仆虫属为单元发生, 且小腔游仆虫与艾美游仆虫(Euplotes amieti)、阔口游仆虫(E. eurystomus)和伍氏游仆虫(E. woodruffi)聚在一起。 关键词：纤毛虫; 小腔游仆虫; 形态学; 细胞发生; 系统学; 核糖体小亚基基因(SSU rDNA)
\end{abstract}

\section{Morphology, ontogeny and molecular phylogeny of Euplotes aediculatus Pierson, 1943 (Ciliophora, Euplotida)}

\author{
Xue Zhang ${ }^{1}$, Yurui Wang ${ }^{1}$, Yangbo Fan ${ }^{1,2}$, Xiaotian $\mathrm{Luo}^{1}$, Xiaozhong $\mathrm{Hu}^{1}$, Feng Gao ${ }^{1 *}$ \\ 1 College of Fisheries, Ocean University of China, Qingdao, Shandong 266003 \\ 2 School of Civil and Environmental Engineering, Harbin Institute of Technology (Shenzhen), Shenzhen, Guangdong 518055
}

\begin{abstract}
Euplotids are the most complex and highly differentiated group of ciliates. In order to further explore the species diversity of euplotids, the morphology and morphogenesis of Euplotes aediculatus, collected from Small West Lake of Qingdao, were investigated using live observations, protargol, and silver nitrate impregnation. Based on the detailed morphological and morphogenetic data, the small subunit ribosomal rDNA (SSU rDNA) was sequenced for E. aediculatus. The species is characterized by the following features: nine frontoventral, five transverse, two left marginal, and two caudal cirri, eight dorsal kineties, and a silverline system of the double-eurystomus type. The main morphogenetic features during division are as follows: (1) the oral primordium of the opisthe develops de novo within a pouch beneath the cortex, the parental adoral zone of membranelles is entirely kept by the proter; (2) fronto-ventral-transverse cirral anlagen (FVTA) II-VI form cirri in the mode of 3:3:3:2:2; (3) frontal cirrus I/1 of the proter and opisthe is generated from the anlage formed de novo; (4) marginal cirri anlagen are formed de novo; (5) the primary dorsal kinety anlagen are from the dedifferentiation of several bristles in the middle of each parental dorsal kinety; (6) the right-most two dorsal kinety anlagen and parent dorsal kineties contribute to two caudal cirri for the proter and the opisthe, respectively. All of these features showed the high conservation of the ontogenetic process in the genus Euplotes. Sequence comparison and phylogenetic analyses based on SSU rDNA revealed a close relationship among Euplotes aediculatus, E. eurystomus, E. amieti, and E. woodruffi, which is consistent with their similar morphology.
\end{abstract}

Key words: ciliates; Euplotes aediculatus; morphology; morphogenesis; phylogeny; small subunit ribosomal rDNA (SSU rDNA) 
纤毛虫原生动物为单细胞真核生物, 种类繁多, 其自由生活的类群广泛分布于海洋、淡水和土壤等 各种生境, 甚至在苔原、极地和高盐等极端生境中 也有发现, 还有一些类群营寄生或共栖生活(Pan et al, 2015; Qu et al, 2015; Dong et al, 2016; Li et al, 2016)。纤毛虫高度分化的细胞器、分司不同功能的 两型核结构以及特有的有性生殖方式(接合生殖), 使其作为重要的模式生物在细胞生物学、遗传学、 生态学以及真核生物的起源和进化等领域具有重 要的研究价值(Corliss, 1979; Lynn, 2008; 宋微波等, 2009)。

游仆类是纤毛虫中进化最为复杂和高等的一 大类群, 其多样性、发生学及系统学一直是国内外 研究的热点(Carter, 1972; Curds, 1975; Shao et al, 2010; Yi et al, 2012)。经典的系统分类中, 游仆类隶 属于纤毛虫中的腹毛类(Kahl，1932；Borror，1972； Corliss, 1979; Borror \& Hill, 1995), 但也有研究认 为, 游仆类与典型的腹毛类均为独立的亚纲级阶元 (Adl et al, 2012; Gao et al, 2016)。游仆虫属(Euplotes) 是游仆类中种类最多的一个属, 目前属内已知物种 70 余种, 广泛分布于海洋、淡水、土壤等各种生境 (Curds, 1975; Curds \& Wu，1983; 宋微波等, 2009; Syberg-Olsen et al, 2016)。本属的基本特征为高度背 腹扁平, 腹面纤毛分化形成额腹棘毛、横棘毛、尾 棘毛等, 以此作为支撑、爬行于基质上; 背面纤毛 退化为触毛, 成列分布; 体表不同程度盔甲化, 背 面常有纵行的肋突结构; 口围带高度发达, 通常占 体长的 $1 / 2$ 以上; 口侧膜单片(Curds, 1975; 宋微波 等, 2009; Jiang et al, 2010b)。本属普遍存在特殊的背 面银线系, 可分为 single-vannus型、 double-eurystomus型、double-patella型、 multiple型和complex 型, 为种间鉴定的重要依据之一(Curds, 1975; 谢冬 梅等, 2016)。种间因具有极相似的形态特征(例如体 型、大小、纤毛图式、皮层结构等)而导致种类划分 相当困难。然而, 诸如额腹棘毛、横棘毛、缘棘毛 及尾棘毛的产生方式及数目在不同种间存在差异, 因而成为种类区分的重要依据。

Pierson (1943)基于美国的多个淡水种群和4个 低盐度咸水(盐度 $2.3 \%$ ) 种群对小腔游仆虫(Euplotes aediculatus)进行了首次报道, 之后, 国外多位学者 对该种进行了研究。由于小腔游仆虫和阔口游仆虫 (E. eurystomus)在体型、纤毛图式、银线系模式等方
面有许多相同之处，长期以来这 2 个种常被混淆。 Pierson等(1968)提供了 2 个种详细的形态学区别特 征，并对以往的报道进行了鉴别分析。庞延斌和魏 红兵(1999)报道了小腔游仆虫上海种群的形态学特 征, 并首次提供了细胞发生学信息, 这是中国种群 的唯一报道。虽然关于小腔游仆虫的研究很多, 但 缺乏在详细的形态学基础上的分子系统学研究。

本工作基于小腔游仆虫青岛小西湖种群的形 态学及发生学研究, 提供了详细的活体特征、纤毛 图式信息、完整的细胞发生时期银染照片及描述; 并在准确的物种鉴定基础上，测定了其核糖体小亚 基基因(SSU rDNA)序列, 通过序列比较和分子系 统树构建等方法对其系统地位进行了分析。

\section{1 材料与方法}

\section{1 样品采集与处理}

本种于 2013 年 10 月 10 日利用直接采水法采自 青岛小西湖, 水温约 $20^{\circ} \mathrm{C}$, 淡水, 湖水较清澈。样品 采集后, 在实验室将水样直接倒入培养皿中, 解剖 镜下去除大型后生动物及多余沉积物, 加入适量米 粒维持细菌繁殖, 以供纤毛虫摄食, 室温 $\left(\right.$ 约 $\left.20^{\circ} \mathrm{C}\right)$ 培养2-3天。

\section{2 形态学和细胞发生学研究}

在解剖镜下观察培养血中虫体的体色、外形(包 括柔软度、伸缩性等)及自由运动方式等; 微吸管挑 取虫体并吸取少量原位水置于载玻片上, 进行活体 压片(Foissner, 1991), 在显微镜下用明视野和微分 干涉观察 (放大倍数100-1,000×) 虫体细节特征, 包 括大小、纤毛器、伸缩泡、表膜皮层结构(有无皮层 颗粒、表膜泡等结构)、射出体、内质及核器等特征。 用蛋白银染色法 (Wilbert，1975) 和银浸染色法 (Foissner, 2014)对虫体进行染色并制成永久制片, 显示其纤毛图式、核器结构及银线系特征。在显微 镜下对虫体各特征进行数据统计, 使用绘图器绘制 虫体纤毛图式及核器。本文所涉及名词及术语参照 宋微波等(2009)。

\subsection{DNA提取、基因扩增与测序}

挑取虫体并用抽滤(滤膜规格 $0.22 \mu \mathrm{m}$ ) 后的原 位水洗净, 使用DNeasy Blood \& Tissue Kit (QIAGEN, Germany)试剂盒提取虫体基因组DNA, 具体操作 步骤参见试剂盒说明书。核糖体小亚基基因(SSU rDNA) 序列的扩增使用真核生物通用引物 $18 \mathrm{~s}-\mathrm{F}$ 
(5'-AAC CTG GTT GAT CCT GCC AGT-3') 和18s-R (5'-TGA TCC TTC TGC AGG TTC ACC TAC-3') (Medlin et al, 1988)。PCR扩增后, 产物直接送测序 公司测序(Yi et al, 2009)。

\section{4 分子系统学分析}

除本文新测序列外, 其余 72 条序列均来自 GenBank数据库。原腹毛亚纲作为外类群。

使用在线服务器 GUIDANCE2 Server (http:// guidance.tau.ac.il/index.html)进行序列比对, 算法选 择MAFFT, 其余参数为默认值。比对之后根据每列 的得分, 将 0.6 以下的位点删除, 并删除两端的引物 序列, 最后剩余 1,958 个位点构建系统树。使用在线 服 务 器 CIPRES Science Gateway (http://www.phylo.org)构建分子系统树。使用在线 软件RAxML-HPC2 v8 on XSEDE (Stamatakis et al, 2008)构建最大似然树 (maximum likelihood, ML), 选择Modeltest v.3.7 (Posada \& Crandall, 1998)筛选 的GTR $+I+G$ 作为最优模型, 节点置信值来自
1,000 叠代重复抽样; 贝叶斯树(Bayesian inference, BI) 使用在线软件 MrBayesian v3.2.6 on XSEDE (Ronquist \& Huelsenbeck, 2003)构建, 选择MrModeltest v.2 (Nylander, 2004)笮选的GTR + I + G作为 最优模型, MCMC 模拟运行两组四条链 10,000,000 代，每100代取样1次，前10,000棵树舍弃，剩余的 树用来计算后验概率。使用MEGA 6.0 (Tamura et al, 2007)调整树形。分类系统参见Gao等(2016)。

\section{2 结果}

\section{1 形态学描述}

活体状态下, 小腔游仆虫虫体腹面观呈卵圆形 至方形，前端较方阔，后端针圆，虫体前端有一非 常透明的领状突起，从虫体右侧 $1 / 4$ 处绕至虫体左 侧 $1 / 4$ 处, 最高处约 $10 \mu \mathrm{m}$; 个体大小较稳定, 长 150-170 $\mu \mathrm{m}$, 宽100-120 $\mu \mathrm{m}$, 厚幅比约为 $1: 3$, 背面 稍隆起, 具6条明显的肋突(背触毛着生), 腹面扁平, 无明显肋突(图1A; 图2A-C)。胞质无色, 虫体口区
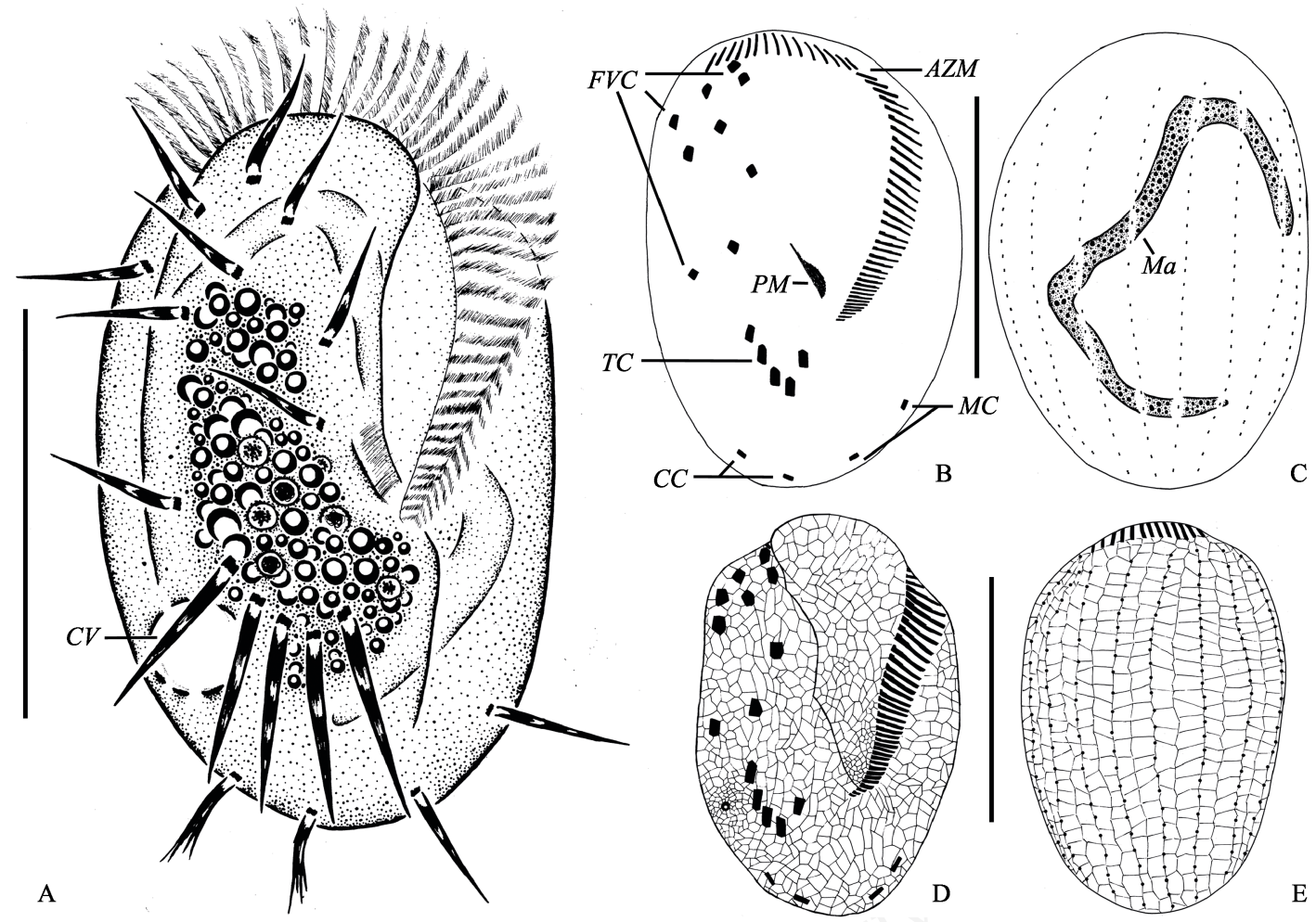

图1 小腔游仆虫的活体、蛋白银染色及银浸法染色的形态。A: 典型个体腹面观; B、C: 纤毛图式腹面(B)和背面(C)观; D、 $\mathrm{E}$ : 腹面(D)和背面(E)银线系。 AZM: 口围带; CC: 尾棘毛; CV: 伸缩泡; FVC: 额腹棘毛; Ma: 大核; MC: 缘棘毛; PM: 口侧 膜; TC: 横棘毛。比例尺: $100 \mu \mathrm{m}$ 。

Fig. 1 Morphology of Euplotes aediculatus in vivo, after protargol and silver nitrate impregnation. A, Ventral view of a representative individual; B and C, Ventral (B) and dorsal (C) view of the infraciliature and nuclear apparatus; D and E, Silverline system on ventral (D) and dorsal side (E); AZM, Adoral zone of membranelles; CC, Caudal cirri; CV, Contractile vacuole; FVC, Frontoventral cirri; $M a$, Macronucleus; $M C$, Marginal cirri; $P M$, Paroral membrane; TC, Transverse cirri. Scale bar $=100 \mu \mathrm{m}$. 

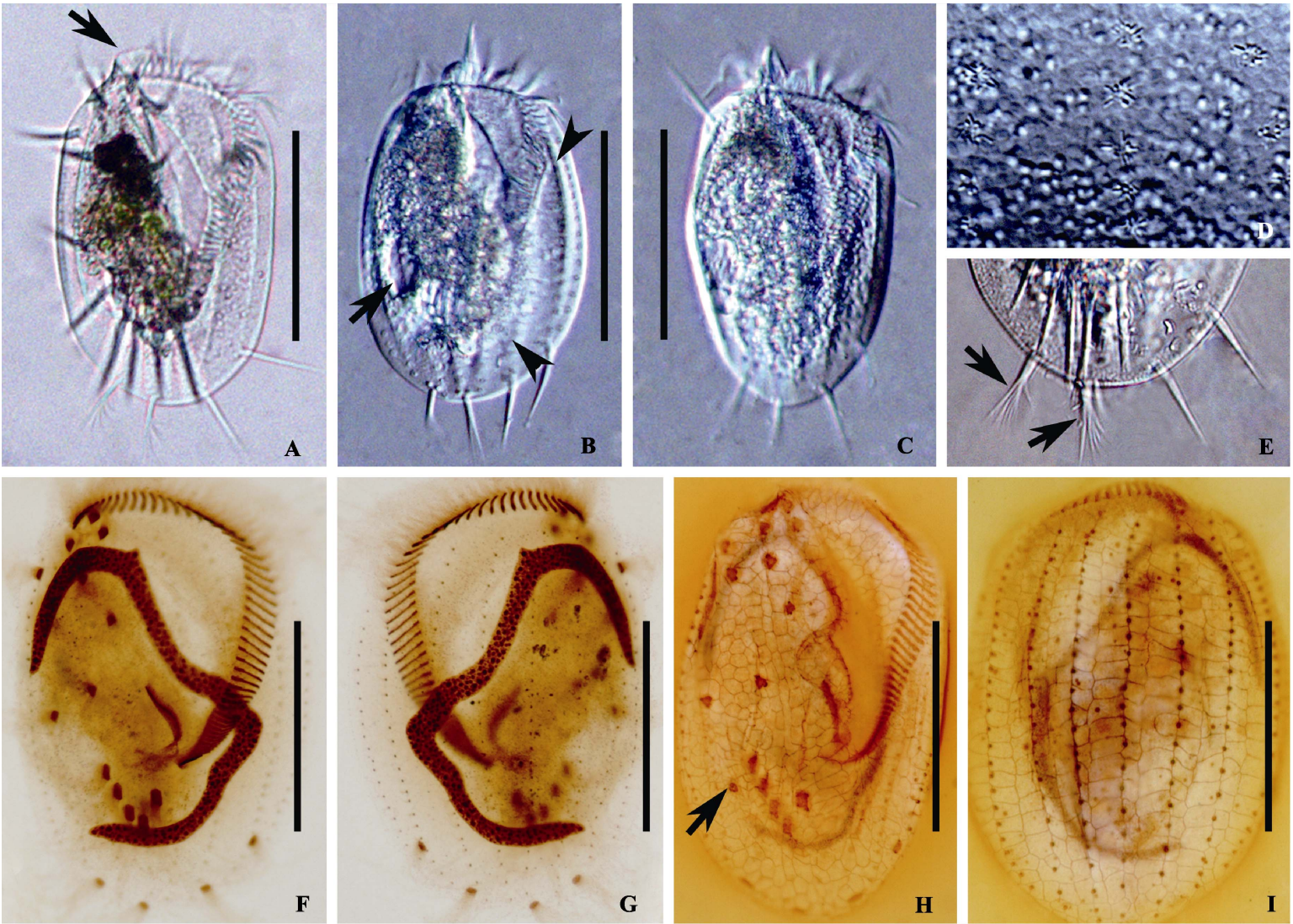

图2 小腔游仆虫的形态结构。A-E: 小腔游仆虫的活体照片; F, G: 蛋白银染色照片; H, I: 银浸法染色照片。A: 典型个体 腹面观，箭头示领状结构; B, C: 不同个体腹面观示不同体型, B图箭头指示伸缩泡, 无尾箭头指示背面肋突; D: 示虫体背面 围绕背触毛的皮层颗粒; E: 虫体后部腹面观，示缘棘毛和分叉的尾棘毛; F, G: 纤毛图式腹面(F)和背面(G)观; H, I: 腹面(H) 和背面(I)银线系, $\mathbf{H}$ 图箭头指示伸缩泡。比例尺: $100 \mu \mathrm{m}$ 。

Fig. 2 Morphology of Euplotes aediculatus. A-E, Photomicrographs in vivo; F and G, Photomicrographs after protargol; H and I, Photomicrographs after silver nitrate impregnation. A, Ventral view of a representative individual, arrow points to collar; B and C, Ventral views, showing different body shapes, arrow in picture B points to contractile vacuole, arrowheads in picture B point to ribs in the back; D, Dorsal view, showing the cortical granules arranged around the dorsal cilia; E, Posterior portion of an individual, showing the caudal cirri (arrows) and the left marginal cirri; F and G, Ventral (F) and dorsal (G) view of the infraciliature and nuclear apparatus; $\mathrm{H}$ and I, Silverline system on ventral $(\mathrm{H})$ and dorsal side $(\mathrm{I})$, arrow in picture $\mathrm{H}$ points to contractile vacuole. Scale bar $=$ $100 \mu \mathrm{m}$

及边缘区域透明，口后中部区域由于含有食物泡和 许多内质颗粒而呈灰黑色(图1A；图2A-C)。背面皮 膜下具无色针棒状皮层颗粒，长约1 $\mu \mathrm{m}, 7-9$ 个为 一组，呈花环状聚在背触毛基部(图2D)。单一伸缩 泡位于最右侧横棘毛下方偏右处(图1A，图2B), 舒 张状态直径约 $25 \mu \mathrm{m}$, 舒张时间约 $90 \mathrm{~s}$, 舒张状态变 为收缩状态只需 $1 \mathrm{~s}$ 。腹面观具一枚镜像“ $C \mathrm{C}$ ”型大核, 左上部有一凹陷。运动方式为典型的游仆类爬行方 式，即依靠腹面棘毛爬行于基质，速度较快。

口区阔大呈倒三角形，约占体长的 $2 / 3$; 口围带 由47-55片小膜构成(表1), 从腹面额腹棘毛前端处 (约3片小膜)绕到背面领状突起处(约12片小膜), 再 绕回腹面并延伸至体长 $2 / 3$ 处, 腹面小膜基本呈平
直排布或稍微向内弯曲; 口侧膜位于口围带末端右 侧口唇下方，由不规则排列的毛基粒组成(图 1B, 图2F)。

棘毛数较恒定(表1)：具9根额腹棘毛； 5 根横棘 毛; 2根左缘棘毛(图1B; 图2F)；2根尾棘毛，且活体 状态下尾棘毛末端纤毛呈分散状(图1A，图2A，E)。 活体状态下, 额腹棘毛及缘棘毛长约 $40 \mu \mathrm{m}$, 横棘 毛较粗壮 (长约 45-50 $\mu \mathrm{m}$ ), 尾棘毛稍短(约 35$40 \mu \mathrm{m}$ )。背触毛恒为 8 列，中间一列具21-26对毛基 粒，最左侧一列具15-23对毛基粒(图1C，图2G，表 1)。背面银线系为double-eurystomus型(图1E，图2I), 腹面银线系可见伸缩泡开口位于最右侧横棘毛的 右侧(图2H)。 
表1 小腔游仆虫青岛小西湖种群的形态学数据。所有数据均来自蛋白银染色制片。

Table 1 Morphometric characterizations of Euplotes aediculatus from Small West Lake of Qingdao based on protargol-stained specimens

\begin{tabular}{|c|c|c|c|c|c|c|c|}
\hline 特征 Character & $\begin{array}{l}\text { 最小值 } \\
\text { Minimum }\end{array}$ & $\begin{array}{l}\text { 最大值 } \\
\text { Maximum }\end{array}$ & $\begin{array}{l}\text { 平均值 } \\
\text { Mean }\end{array}$ & $\begin{array}{l}\text { 中值 } \\
\text { Median }\end{array}$ & $\begin{array}{l}\text { 标准差 } \\
\text { Standard } \\
\text { deviation }\end{array}$ & $\begin{array}{l}\text { 变异系数 } \\
\text { Coefficient } \\
\text { of variation }\end{array}$ & $\begin{array}{l}\text { 个体数 } \\
\text { Number } \\
\text { of cells }\end{array}$ \\
\hline 体长 Length of body $(\mu \mathrm{m})$ & 115 & 142 & 128.3 & 129 & 7.25 & 5.7 & 20 \\
\hline 体宽 Width of body $(\mu \mathrm{m})$ & 76 & 101 & 92.2 & 93.5 & 6.28 & 6.8 & 20 \\
\hline 口区长 Length of adoral zone $(\mu \mathrm{m})$ & 79 & 98 & 86.3 & 86.5 & 4.74 & 5.5 & 20 \\
\hline 口围带小膜数目 Number of adoral membranelles & 47 & 55 & 51.1 & 51.5 & 1.90 & 3.7 & 20 \\
\hline 额腹棘毛数目 Number of frontoventral cirri & 9 & 9 & 9 & 9.0 & 0 & 0 & 19 \\
\hline 横棘毛数目 Number of transverse cirri & 5 & 5 & 5.0 & 5.0 & 0 & 0 & 20 \\
\hline 缘棘毛数目 Number of marginal cirri & 2 & 2 & 2.0 & 2.0 & 0 & 0 & 20 \\
\hline 尾棘毛数目 Number of caudal cirri & 2 & 2 & 2.0 & 2.0 & 0 & 0 & 20 \\
\hline 背触毛列数 Number of dorsal kineties & 8 & 8 & 8.0 & 8.0 & 0 & 0 & 20 \\
\hline $\begin{array}{l}\text { 中央背触毛列毛基体数目 } \\
\text { Number of dikinetids in mid-dorsal kinety }\end{array}$ & 21 & 26 & 21.9 & 21.0 & 1.36 & 6.2 & 20 \\
\hline $\begin{array}{l}\text { 最左侧背触毛列毛基体数目 } \\
\text { Number of dikinetids in the leftmost dorsal kinety }\end{array}$ & 15 & 23 & 18.5 & 18.0 & 1.73 & 9.4 & 20 \\
\hline
\end{tabular}

\section{2 细胞发生学描述}

\subsection{1 口器}

口器发生起始于后仔虫(细胞横二分裂时位于 分裂沟后部的子代，与前仔虫相对)口原基的出现， 后仔虫的口原基独立形成于老口围带下方皮膜深 层的拿腔内, 最初为一团无序的毛基粒群(图3A)。 随着毛基粒的快速增殖，龛腔增大，口原基开始从 前向后组装成新的小膜并逐渐向表层延伸(图3A-C, $\mathrm{E}, \mathrm{F})$ 。最终扩展至皮膜表层, 并向右弯折形成后仔 虫的口围带(图3H)。

后仔虫的波动膜原基于皮膜深层的龛腔内形成 于口原基末端附近, 并随着细胞发生的进行, 逐渐增 殖, 最终发育为后仔虫的波动膜(图3B, C, E, F, H)。 整个细胞发生过程中, 老口围带和波动膜均完全保 留, 由前仔虫继承(图3E, H)。

\subsection{2 腹面棘毛}

口原基形成的同时，口区右侧横棘毛前部区域 独立产生两组各有 5 列短的由疏松毛基粒形成的线 状额-腹-横棘毛原基(自左至右为原基II-VI), 但由 于时期的缺乏, 我们无法判断是否为次级发生型 (图3A)。随着毛基粒的增殖, 每列原基逐渐变粗, 并 开始发生断裂，5列额-腹-横棘毛原基列以 3:3:3:2:2 的模式断裂(图3B, C, E, F)。最后新形成的棘毛迁移 至最终位置, 形成前后仔虫的腹面棘毛: 原基II-VI 各贡献最下方的 1 根棘毛共形成 5 根横棘毛, 其余 8 根棘毛均为额腹棘毛(图3H)。
额-腹-横棘毛原基断裂时，在皮膜表层前仔虫 额-腹-横棘毛原基及老口侧膜左上方独立产生一 组毛基粒，此为前仔虫最左侧额-腹-横棘毛原基 I; 相应的，后仔虫的最左侧额-腹-横棘毛原基I独立 产生于后仔虫波动膜原基的左上方(图3B, C, E, F)。 随后，两组原基增殖并迁移至其最终位置，形成前 后仔虫新的最左侧额腹棘毛(图3H)。

\subsection{3 缘棘毛}

当额-腹-横棘毛原基开始形成片段的时候，前 后仔虫的缘棘毛原基分别于老口围带和后仔虫口 原基末端左侧附近独立形成(图3B)。之后，两组原 基逐渐增殖变粗, 并最终断裂形成前后仔虫的两根 缘棘毛(图3C, E, F, H)。

\subsection{4 背触毛及尾棘毛}

背触毛原基于每一列老背触毛列的中线处由 老结构反分化形成, 为典型的初级发生模式(图 3D)。随后，背触毛原基分成前后两组，分别向前后 两端迁移, 形成前后仔虫的背触毛列, 并最终替代 老结构(图3H)。

前仔虫最右侧两列背触毛原基末端各形成 1 根 尾棘毛, 后仔虫最右侧两列老背触毛末端各形成 1 根尾棘毛, 并随着背触毛列向两端移动, 迁移至最 终位置(图3G)。

\subsection{5 核器}

大核在细胞发生过程中, 逐渐由左上部有一匹 陷的镜像“C”型形成短棒状(图3 $\mathrm{E}, \mathrm{F})$; 至细胞发生 

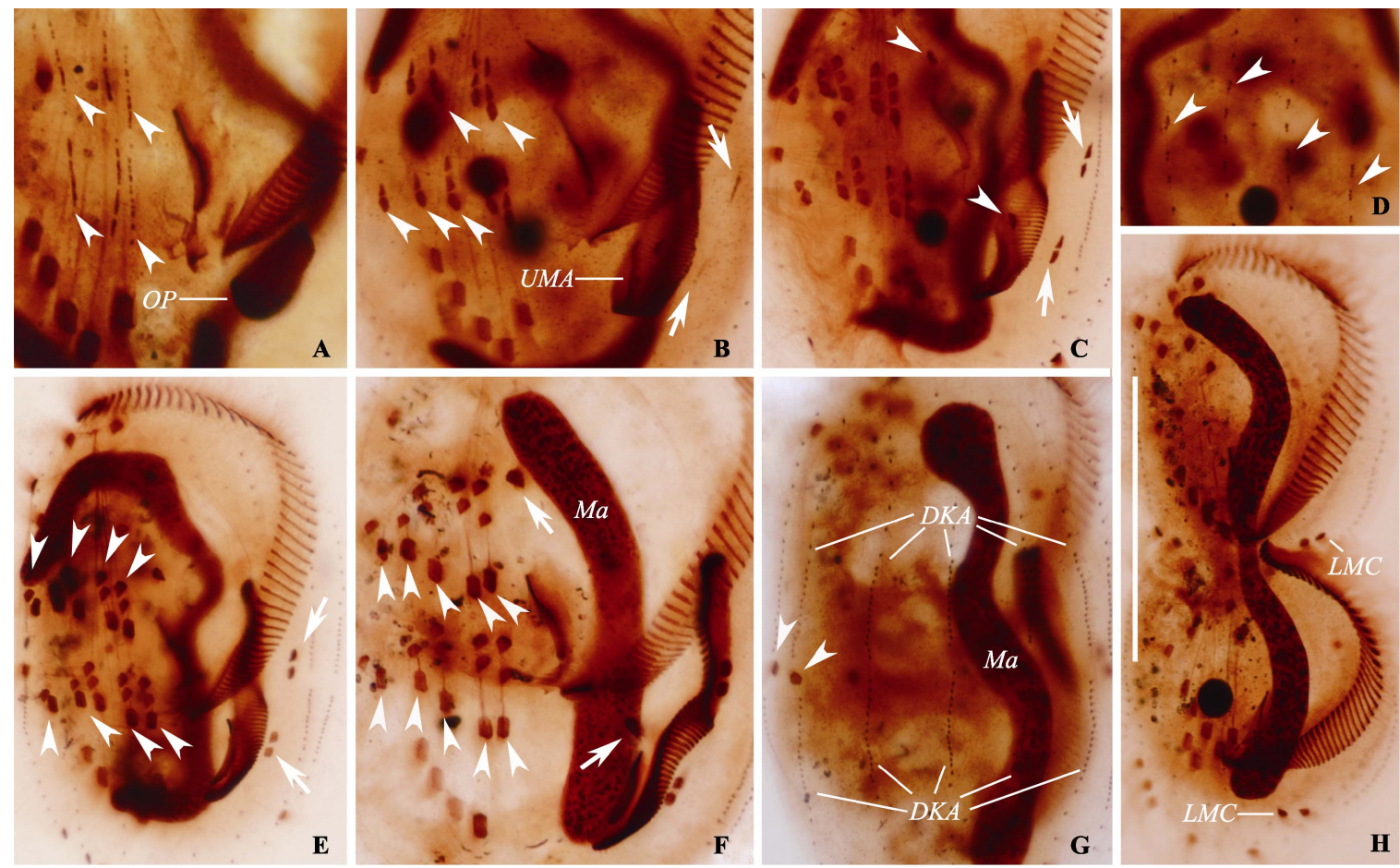

图3 小腔游仆虫细胞发生个体银染制片的显微照片。A: 发生早期个体, 示后仔虫口原基出现, 短箭头示前后仔虫的额-腹一 横棘毛原基; B: 发生早中期个体腹面观, 短箭头示额-腹一横棘毛原基增粗并开始发生断裂, 长箭头示前后仔虫的缘棘毛原 基; C: 发生中期个体腹面观, 示额-腹一横棘毛原基断裂形成棘毛, 短箭头示前后仔虫的最左侧一根额腹棘毛, 长箭头示缘棘 毛原基开始断裂形成缘棘毛; D: 发生早中期个体背面观, 短箭头示新形成的背触毛原基; E: 发生中期个体腹面观, 短箭头 示额一腹一横棘毛片段化基本完成并开始迁移，长箭头示前后仔虫新的缘棘毛; F: 发生后期个体腹面观, 短箭头示前后仔虫的 横棘毛, 长箭头示最左侧一根额腹棘毛, 此时大核呈棒状; G: 发生后期个体背面观, 短箭头示前仔虫新形成的尾棘毛; H: 发生末期个体腹面观, 示新形成棘毛基本迁移至其最终位置, 大核开始发生分裂。DKA: 背触毛原基; LMC: 左缘棘毛; Ma: 大核; OP: 后仔虫口原基; UMA：波动膜原基。比例尺: $70 \mu \mathrm{m}$ 。

Fig. 3 Photomicrographs of Euplotes aediculatus during morphogenesis after protargol impregnation. A, Ventral view of an early divider, showing the oral primordium in opisthe, arrowheads mark the frontal-ventral-transverse cirral streaks of both dividers; B, Ventral view of an early-middle divider, arrowheads show the differentiation of frontal-ventral-transverse cirral anlagen, arrows marks the marginal anlagen; C, Ventral view of a mid-stage divider, arrowheads demonstrate the leftmost frontal cirrus (I/1) in both proter and opisthe, arrows marks the differentiation of marginal anlagen; D, Dorsal view of an early-middle divider, arrowheads show the formation of dorsal kineties anlagen; E, Ventral view of another mid-stage divider, arrowheads show the differentiation of frontal-ventral-transverse cirral anlagen almost complete, arrows point to the new marginal cirri of both daughter cells; F, Ventral view of a late divider, noting the macronucleus become a short strand, arrowheads show the transverse cirri, arrows mark the leftmost frontal cirri (I/1); G, Dorsal view of another late divider, arrowheads show the newly formed caudal cirri in the proter; H, Ventral view of a last stage divider, showing cirri almost in their final position and the division of the macronucleus. DKA, Dorsal kineties anlagen; LMC, Left marginal cirri; Ma, Macronucleus; OP, Opisthe's oral primordium; UMA, Undulating membrane anlagen. Scale $\mathrm{bar}=70 \mu \mathrm{m}$.

末期，大核分裂，分别在前后仔虫中形成新的大核 (图3G, H)。

\section{3 分子系统学分析}

小腔游仆虫青岛小西湖种群的SSU rDNA序列 已提交至GenBank数据库, 序列号为KY921848。该 序列包含 1,809 个碱基, $\mathrm{G}+\mathrm{C}$ 含量为 $44.44 \%$ 。

我们选择GenBank中有SSU rDNA序列信息的 游仆目 5 个科(楯纤虫科、舍太虫科、游仆虫科、腹 棘虫科和尾刺虫科)的代表类群, 并以原腹毛亚纲
作为外类群构建了系统进化树(图4)。结果显示: (1) 以游仆虫属为代表的游仆虫科为明确的单源发生 $(100 \% \mathrm{ML}, 1.00 \mathrm{BI}) ;(2)$ 来自 GenBank的小腔游仆虫 的 6 个种群与阔口游仆虫(AF452707) 以满分置信值 聚为一支, 之后与本工作新测序的小腔游仆虫种群 聚为姐妹支 $(100 \% \mathrm{ML}, 1.00 \mathrm{BI})$ 。序列比对结果显示, 本工作新测序的小腔游仆虫的SSU rDNA序列与姐 妹支内各种群序列最少相差19个碱基, 最多相差 29 个碱基(图5)。 


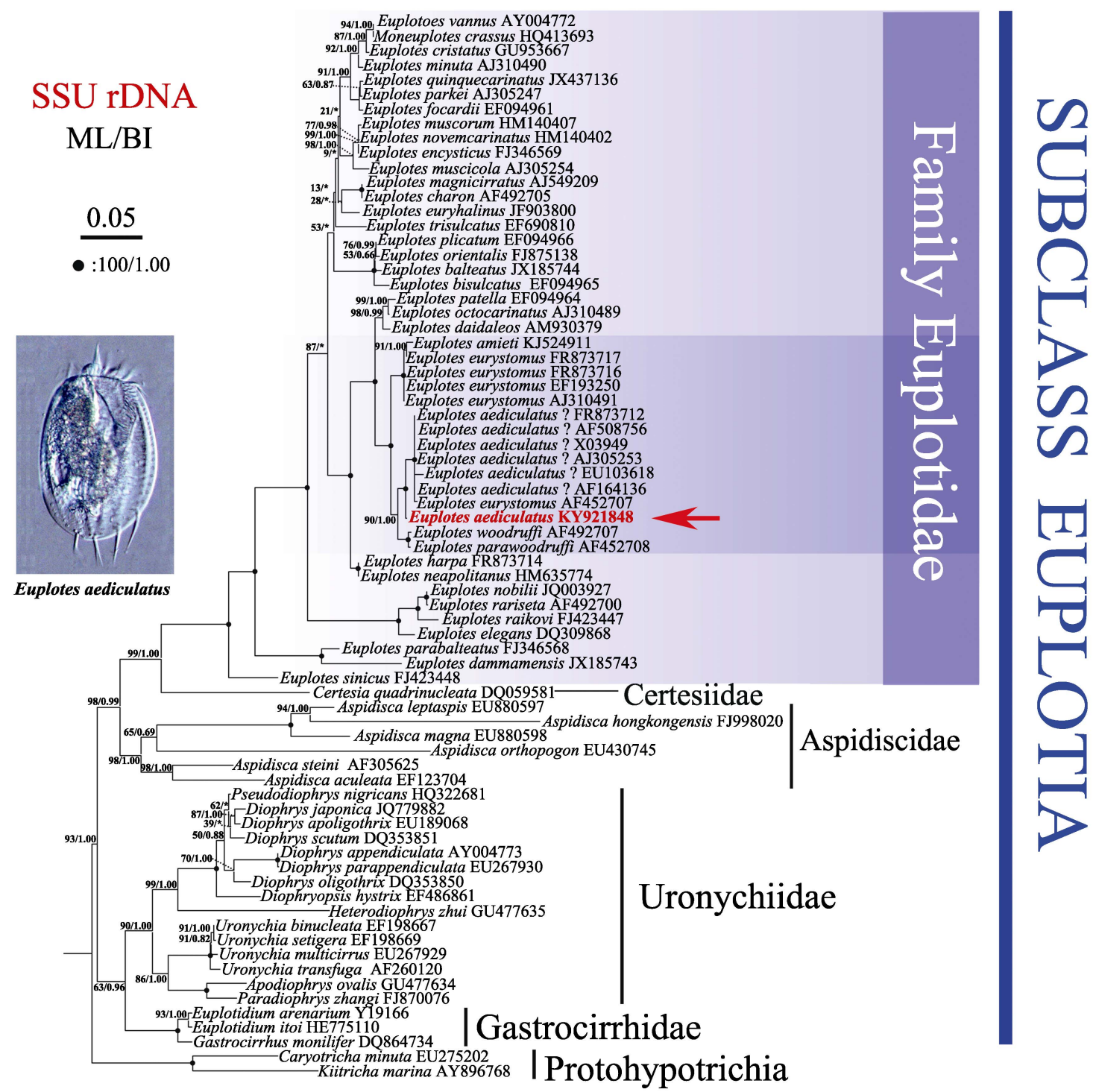

图4 基于核糖体小亚基基因序列构建的最大似然树。本文新测序列加粗显示。节点置信值以最大似然树/贝叶斯树顺序给出。 黑点表示满分置信值(100/1.00), “*”表示贝叶斯树与最大似然树拓扑结构不同。比例尺代表每100个碱基位点中有5个碱基替代。 Fig. 4 Maximum likelihood (ML) tree based on small subunit ribosomal DNA sequences. Newly characterized sequence in this study is shown in bold. The numbers at the nodes represent the support values of ML/Bayesian inference (BI). Fully supported $(100 / 1.00)$ branches are marked with solid circles. Asterisks reflect the disagreement between ML and BI. All branches are drawn to scale. The scale bar corresponds to five substitutions per 100 nucleotide positions.

Euplotes eurystomus AF452707
Euplotes aediculatus EU103618
Euplotes aediculatus FR873712
Euplotes aediculatus X03949
Euplotes aediculatus AJ305253
Euplotes aediculatus AF164136
Euplotes aediculatus AF508756
Euplotes aediculatus XX000000

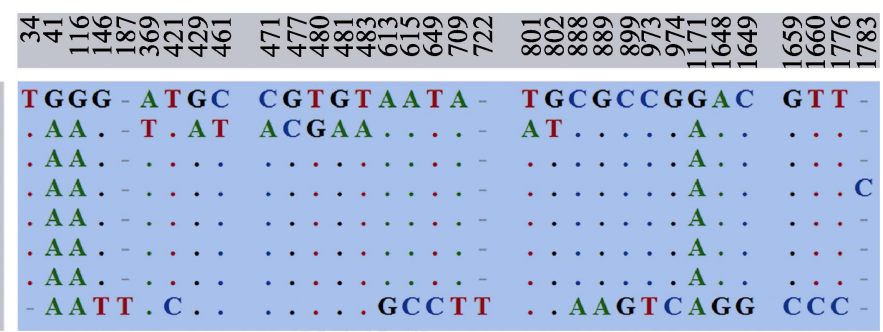

图5 7个Euplotes aediculatus种群及 1 个E. eurystomus种群的SSU rDNA碱基差异比较。数字代表比对后的碱基所在位置, 缺 失位点用(-)表示, 比对一致的碱基用(.)表示。

Fig. 5 Unmatched sites from SSU rDNA sequence alignment of seven Euplotes aediculatus populations and one E. eurystomus population. Numbers indicate the positions of nucleotides in the alignment, missing sites are represented by dashes (-), and matching sites are marked with dots (.). 


\section{3 讨论}

\section{1 形态学比较}

3.1.1 小腔游仆虫青岛小西湖种群与其他种群的 形态学比较

小腔游仆虫是淡水生境中常见的游仆虫种类 之一, 多位学者对该种进行了报道(Pierson, 1943; Tuffrau, 1960; Pierson et al, 1968; Carter, 1972; 庞延 斌和魏红兵, 1999)。本文对小腔游仆虫青岛种群给 出了首次报道, 青岛小西湖种群的主要形态学特征 (体型卵圆形, 腹面无肋突, 背触毛着生于 6 列较明 显的脊上; 口区右缘明显的小腔; 镜像“ $C$ ”型大核; 纤毛图式)与模式种群完全一致, 因此将本种群鉴 定为小腔游仆虫。

本种群与形态描述较全的几个种群的腹面棘 毛模式、背面银线系等特征也都高度一致, 种群间 的区别主要如下：与Pierson等(1968)报道的美国马 里兰种群和美国华盛顿种群相比, 本种群具更多口 围带小膜(47-55片 vs. 约40片); 庞延斌和魏红兵 (1999)报道了上海种群并提供了细胞发生信息, 本 种群与上海种群的区别在于个体大小 (150-170 $\mu \mathrm{m}$ $\times 100-120 \mu \mathrm{m}$ vs. $120-130 \mu \mathrm{m} \times 80-85 \mu \mathrm{m})$ 及口围 带小膜数(47-55片 vs. 40-50片)。前人的研究认为 Tuffrau (1960)以及Carter (1972)报道的以阔口游仆 虫为名的种群, 实际均为小腔游仆虫(Pierson et al, 1968; Curds, 1975; 庞延斌和魏红兵, 1999)。本种群 与 Carter种群的主要区别在于本种群个体稍大 (150-170 $\mu \mathrm{m} \times 100-120 \mu \mathrm{m}$ vs. $121.5-135 \mu \mathrm{m} \times$ 73.5-85.5 $\mu \mathrm{m})$, 但中间一列背触毛毛基粒较少 (21-26 vs. 28-31), 且Carter报道的3个种群中, 只有 1 个种群背触毛恒为 8 列, 与我们的种群相同, 另外 2 个种群背触毛则恒为 9 列。虽然本研究的种群与前 人研究的种群个体大小、口围带小膜数目稍有差异, 但均属于种群间差异, 因此为同种。

\subsection{2 与属内相近种的形态学比较}

20 世纪初期以前，作者仅以体型大小、背面的 脊、腹面棘毛的排列做为游仆虫的分类依据(Kahl, 1932)。之后, 蛋白银染色方法和银浸染色方法的应 用使得额腹棘毛数目、口围带形状和小膜数目、背 触毛列数、大核形状和银线系成为重要的分类标准 (Tuffrau, 1960; Carter, 1972; Curds, 1975)。由于早期 研究缺乏详细的棘毛数目、银线系模式等形态学信
息，并且一些种有相同或相似的体型、大小、额腹 棘毛数目、背触毛列数等特征, 这些都使得游仆虫 属的分类存在极大的混乱和困难。目前, 属内已知 种多达 70 余种 (Tuffrau，1960; Curds，1975; Syberg-Olsen et al, 2016)。形态特征的不同组合形成了 游仆虫不同程度的种间差异, 其中额腹棘毛数目和 银线系类型是非常重要且稳定的种间区分依据。除小 腔游仆虫之外, 具9根额腹棘毛和double-eurystomus 型银线系的淡水生游仆虫还有 4 种：艾美游仆虫 (Euplotes amieti)、阔口游仆虫、伍氏游仆虫 $(E$. woodruffi $)$ 和芬克游仆虫(E. finki)。小腔游仆虫具以 下特征可与这4种明显区分(表2): (1)活体状态和染 色后呈现的纤毛图式口围带均呈垂直或平滑弧形, 而阔口游仆虫和艾美游仆虫为“ $\mathrm{S}$ ”型—腹面口围 带有弯曲; (2)背面具6列明显肋突, 而阔口游仆虫 和艾美游仆虫无明显肋突; (3)背触毛列数8列, 而 阔口游仆虫具 8-12列，艾美游仆虫具 12-15列，伍 氏游仆虫具9-10列, 芬克游仆虫具7列; (4)大核形 状镜像“C $\mathrm{C}$ ”型且左上部有一凹陷，而阔口游仆虫“3” 字型，伍氏游仆虫为“ $T$ ”或“ $Y$ ”型，芬克游仆虫为 “C”到“J”型(Curds, 1975; Foissner, 1982; Dragesco, 2003; Dai et al, 2013; Liu et al, 2015)。

\section{2 细胞发生学比较}

小腔游仆虫的形态学报道虽然较多, 但是仅 庞延斌和魏红兵(1999)的上海金山卫种群提供了 发生学信息。根据对小腔游仆虫青岛小西湖种群无 性生殖期间形态发生的跟踪观察, 本研究结果与 庞延斌和魏红兵(1999)报道的上海金山卫种群的 细胞发生过程一致, 这说明小腔游仆虫具有稳定 的发生学特征。

游仆虫属内大约有 30 个种有发生学报道, 属内 发生模式有较高的稳定性, 即: 老的口围带和口侧 膜完全被保留, 由前仔虫继承; 后仔虫口侧膜原基 来自于口原基(深层); 前后仔虫的第 1 额腹棘毛均 来源于独立发生的原基; 左缘棘毛原基独立发生等 (Washburn \& Borror, 1972; Ruffolo, 1976; Shao et al, 2010; Fotedar et al, 2016)。小腔游仆虫细胞发生过程 符合以上典型的游仆虫属发生模式。但是, 属内各 种之间的发生学特征也存在细微差异。Shao等(2010) 根据额腹棘毛、横棘毛的形成模式及数目排布将游 仆虫属的发生类型分为5类: raikovi型、orientalis型、 eurystomus型、affinis型和charon型，根据尾棘毛的 


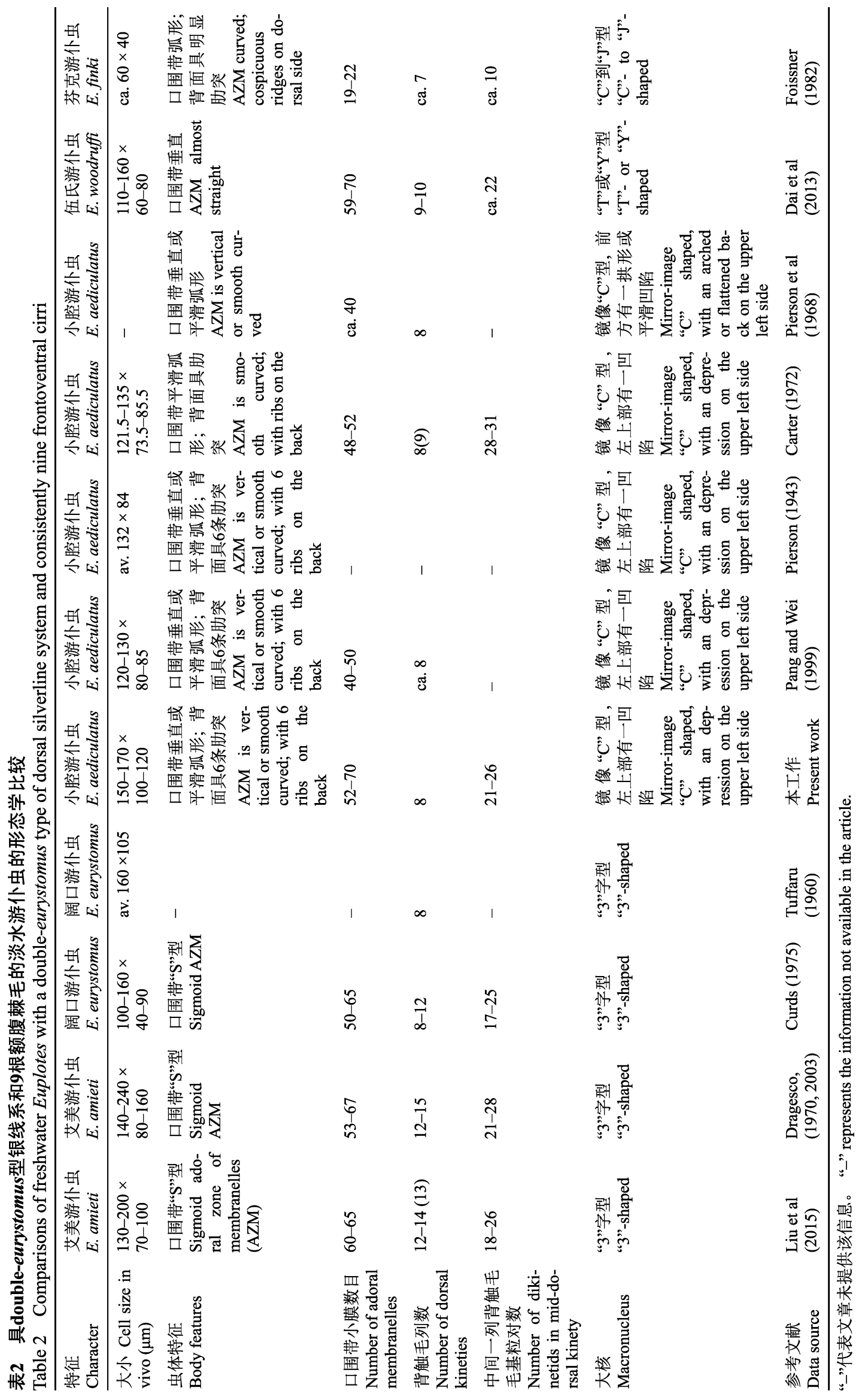


形成方式将发生类型分为3类: focardii型、vannus型 和charon型。而小腔游仆虫额-腹-横棘毛原基的形 成模式为典型的 eurystomus 型, 即 FVT 原基以 3:3:3:2:2方式断裂, 最终以 9 根额棘毛、5根横棘毛 (棘毛 $V I / 2$ 在 $V / 2$ 稍后方) 模式排布，尾棘毛形成类型 为大多数种的focardii型, 即前、后仔虫的最右侧 2 列背触毛列原基后方各产生 1 根尾棘毛, 形成的尾 棘毛数量恒定。左缘棘毛原基独立产生后两组原基 逐渐增殖变粗, 并最终断裂形成前后仔虫的 2 根缘 棘毛, 而不同于该属内部分种类, 例如: 稀毛游仆 虫(Euplotes rariseta)仅产生 1 根缘棘毛( Ma et al, 2008)。

\section{3 系统学比较}

Borror和Hill (1995)根据皮层结构 (银线系类 型)、内共生体、统计学数据以及发生学模式等将游 仆虫属分为 4 个属: Euplotes、Euplotopsis、Euplotoides 和Moneuplotes。但是此划分受到随后的系统学研究 结果的质疑(Yi et al, 2009; Jiang et al, 2010a; Di Giuseppe et al, 2014; Chen et al, 2015), 因此有研究 者建议将 4 个属恢复到原来的单一的游仆虫属( Liu et al, 2015), 本工作的分子系统分析也支持此观点。

基于SSU rDNA序列信息构建的分子系统树显 示, 小腔游仆虫与艾美游仆虫、阔口游仆虫、伍氏 游仆虫聚在一起。这与它们的形态学数据是一致的, 即都是具 9 根额腹棘毛和double-eurystomus型银线 系的淡水生游仆虫。但是目前已有分子信息的阔口 游仆虫和小腔游仆虫若干种群均没有各自聚为一 支(图4)。形态学方面, 小腔游仆虫和阔口游仆虫在 个体大小、体型、纤毛图式、背面银线系模式等方 面有许多相同之处, 因此, 长期以来这两个种常被 混淆，如前文提及的Tuffrau (1960)以及Carter (1972) 将小腔游仆虫错误地报道为阔口游仆虫。Pierson等 (1968)提供的详细形态学资料证明了小腔游仆虫和 阔口游仆虫的区别: (1) 口围带小膜形状(垂直或平 滑弧形 vs. 明显“S”型一腹面口围带有弯曲); (2)领 状结构(不明显 Vs. 明显); (3)背面特征(具6列明显 肋突 vs. 无明显肋突); (4)口围带后缘与中间一根 横棘毛的距离(较近 vs. 较远); (5)大核形状(镜像 “C”型，左上部有一凹陷 vs. “3”字型)。Curds (1975)、庞延斌和魏红兵(1999)也对这2种的区别做 了描述。

本工作涉及的来自GenBank的6条小腔游仆虫
序列, 分别来自系统学分析或者共生关系研究 (Sogin et al, 1986; Petroni et al, 2002; Hewitt et al, 2003; Vannini et al, 2012)。这6个种群均和阔口游仆 虫(AF452707)聚为一支, SSU rDNA序列比较显示 它们与阔口游仆虫(AF452707)分别相差3个碱基、 4 个碱基和 13 个碱基，而与我们的种群相差19-29个 碱基(图5)。鉴于该6个种群都没有发表对应的形态 学信息, 且小腔游仆虫和阔口游仆虫非常容易被混 淆, 因此分析认为: (1)该6个种群可能是错误鉴定 的阔口游仆虫(FR873712、AJ305253、AF164136、 AF508756、X03949)或者其他未知种(EU103618); (2) 如果该 6 个种群与本研究中的小腔游仆虫种群在形 态上不能区分, 则有可能小腔游仆虫存在隐匿种 (Lahr et al, 2014)。很遗憾的是, GenBank中的所有阔 口游仆虫种群也没有对应的形态学信息, 增加了对 这 2 个相似种进行比较的难度, 未来基于完整的形 态学信息的阔口游仆虫的SSU rDNA的分子信息将 有助于解决此混乱。

致谢：感谢宋微波教授对本文构思和成文的帮助！

\section{参考文献}

Adl SM, Simpson AG, Lane CE, Lukes J, Bass D, Bowser SS, Brown MW, Burki F, Dunthorn M, Hampl V, Heiss A, Hoppenrath M, Lara E, Le Gall L, Lynn DH, McManus H, Mitchell EA, Mozley-Stanridge SE, Parfrey LW, Pawlowski J, Rueckert S, Shadwick L, Schoch CL, Smirnov A, Spiegel FW (2012) The revised classification of eukaryotes. Journal of Eukaryotic Microbiology, 59, 429-493.

Borror AC (1972) Revision of the order Hypotrichida (Ciliophora, Protozoa). Journal of Protozoology, 19, 1-23.

Borror AC, Hill BF (1995) The order Euplotida (Ciliophora): taxonomy, with division of Euplotes into several genera. Journal of Eukaryotic Microbiology, 42, 457-466.

Carter HP (1972) Infraciliature of eleven species of the genus Euplotes. Transactions of the American Microscopical Society, 91, 466-492.

Chen XM, Zhao Y, Al-Farraj SA, Saleh AQ, El-Serehy HA, Shao C, Al-Rasheid KAS (2015) Taxonomic descriptions of two marine ciliates, Euplotes dammamensis n. sp. and Euplotes balteatus (Dujardin, 1841) Kahl, 1932 (Ciliophora, Spirotrichea, Euplotida), collected from the Arabian Gulf, Saudi Arabia. Acta Protozoologica, 52, 73-87.

Corliss JO (1979) The Ciliated Protozoa: Characterization, Classification and Guide to the Literature. Pergamon Press, Oxford.

Curds RC (1975) A guide to the species of Euplotes (Hypotri- 
chida, Ciliatea). Bulletin of the British Museum (Natural History) Zoology, 28, 3-61.

Curds RC, Wu ICH (1983) A review of the Euplotidae (Hypotrichida, Ciliophora). Bulletin of the British Museum (Natural History) Zoology, 44, 191-247.

Dai RH, Xu KD, He YY (2013) Morphological, physiological, and molecular evidences suggest that Euplotes parawoodruffi is a junior synonym of Euplotes woodruffi (Ciliophora, Euplotida). Journal of Eukaryotic Microbiology, 60, 70-78.

Di Giuseppe G, Erra F, Frontini F, Dini F, Vallesi A, Luporini $P$ (2014) Improved description of the bipolar ciliate, Euplotes petzi, and definition of its basal position in the Euplotes phylogenetic tree. European Journal of Protistology, 50, 402-411.

Dong JY, Lu XT, Shao C, Huang J, Alrasheid KA (2016) Morphology, morphogenesis and molecular phylogeny of a novel saline soil ciliate, Lamtostyla salina n. sp. (Ciliophora, Hypotricha). European Journal of Protistology, 56, 219-231.

Dragesco J (1970) Ciliés Libres du Cameroun. Annales de la Faculté des Sciences (Numéro hors série). Université Fédérale du Cameroun, Yaoundé. (in French)

Dragesco J (2003) Infraciliature et morphometrie de vingt espèces de ciliés hypotriches recoltés au Rwanda et Burundi, comprenant Kahliella quadrinucleata n. sp., Pleurotricha multinucleata $\mathrm{n}$. sp. et Laurentiella bergeri n. sp. Travaux du Muséum National d'Histoire Naturelle "Grigore Antipa", 45, 7-59. (in French)

Foissner W (1982) Ecology and taxonomy of the Hypotrichida (Protozoa: Ciliophora) of some Austrian soils. Archiv Für Protistenkunde, 126, 19-143. (in French with English abstract)

Foissner W (1991) Basic light and scanning electron microscopic methods for taxonomic studies of ciliated protozoa. European Journal of Protistology, 27, 313-330.

Foissner W (2014) An update of 'basic light and scanning electron microscopic methods for taxonomic studies of ciliated Protozoa'. International Journal of Systematic and Evolutionary Microbiology, 64, 271-292.

Fotedar R, Stoeck T, Filker S, Fell JW, Agatha S, Al Marri M, Jiang JM (2016) Description of the halophile Euplotes qatarensis nov. spec. (Ciliophora, Spirotrichea, Euplotida) isolated from the hypersaline Khor Al-Adaid Lagoon in Qatar. Journal of Eukaryotic Microbiology, 63, 578-590.

Gao F, Warren A, Zhang QQ, Gong J, Miao M, Sun P, Xu DP, Huang J, Yi ZZ, Song WB (2016) The all-data-based evolutionary hypothesis of ciliated protists with a revised classification of the phylum Ciliophora (Eukaryota, Alveolata). Scientific Reports, 6, 24874.

Hewitt EA, Müller KM, Cannone J, Hogan DJ, Gutell R, Prescott DM (2003) Phylogenetic relationships among 28 spirotrichous ciliates documented by rDNA. Molecular Phylogenetics and Evolution, 29, 258-267.

Jiang JM, Zhang QQ, Hu XZ, Shao C, Al-Rasheid KAS, Song
WB (2010a) Two new marine ciliates, Euplotes sinicus sp. nov. and Euplotes parabalteatus sp. nov., and a new small subunit rRNA gene sequence of Euplotes rariseta (Ciliophora, Spirotrichea, Euplotida). International Journal of Systematic and Evolutionary Microbiology, 60, 1241-1251.

Jiang JM, Zhang QQ, Warren A, Al-Rasheid KAS, Song WB (2010b) Morphology and SSU rRNA gene-based phylogeny of two marine Euplotes species, E. orientalis spec. nov. and E. raikovi (Ciliophora, Euplotida). European Journal of Protistology, 46, 121-132.

Kahl A (1932) Urtiere oder Protozoa. I: Wimpertiere oder Ciliata (Infusoria), 3. Spirotricha. Tierwelt Dtl., 25, 399650. (in German)

Lahr DJG, Laughinghouse HD, Oliverio A, Gao F, Katz LA (2014) How discordant morphological and molecular evolution among microorganisms can revise our notions of biodiversity on Earth. Bioessays, 36, 950-959.

Li LQ, Zhao XL, Ji DD, Hu XZ, Al-Rasheid KA, Al-Farraj SA, Song WB (2016) Description of two marine amphisiellid ciliates, Amphisiella milnei (Kahl, 1932) Horváth, 1950 and A. sinica sp. nov. (Ciliophora: Hypotrichia), with notes on their ontogenesis and SSU rDNA-based phylogeny. European Journal of Protistology, 54, 59-73.

Liu MJ, Fan YB, Miao M, Hu XZ, Al-Rasheid KAS, Al-Farraj SA, Ma HG (2015) Morphological and morphogenetic redescriptions and SSU rRNA gene-based phylogeny of the poorly-known species Euplotes amieti Dragesco, 1970 (Ciliophora, Euplotida). Acta Protozoologica, 54, 171.

Lynn DH (2008) The Ciliated Protozoa: Characterization, Classification and Guide to the Literature, 3rd edn. Springer-Verlag, Dordrecht.

Ma HG, Jiang JM, Hu XZ, Shao C, Song WB (2008) Morphology and morphogenesis of the marine ciliate, Euplotes rariseta (Ciliophora, Euplotida). Acta Hydrobiologica Sinica, 32, 57-62.

Medlin L, Elwood HJ, Stickel S, Sogin ML (1988) The characterization of enzymatically amplified eukaryotic 16S-like rRNA-coding regions. Gene, 71, 491-499.

Nylander JA (2004) MrModeltest 2. 2. Department of Systematic Zoology, Evolutionary Biology Centre, Uppsala University, Uppsala.

Pan HB, Hu JX, Jiang JM, Wang LQ, Hu XZ (2015) Morphology and phylogeny of three Pleuronema species (Ciliophora, Scuticociliatia) from Hangzhou Bay, China, with description of two new species, $P$. binucleatum n. sp. and $P$. parawiackowskii n. sp. Journal of Eukaryotic Microbiology, 63, 287-298.

Pang YB, Wei HB (1999) Studies on the morphology and morphogenesis in Euplotes aediculatus. Journal of East China Normal University (Natural Science), (1), 103-109. (in Chinese with English abstract) [庞延斌, 魏红兵 (1999) 小腔游仆虫Euplotes aediculatus形态和形态发生的研究. 华东师范大学学报(自然科学版), (1), 103-109.] 
Petroni G, Dini F, Verni F, Rosati G (2002) A molecular approach to the tangled intrageneric relationships underlying phylogeny in Euplotes (Ciliophora, Spirotrichea). Molecular Phylogenetics and Evolution, 22, 118-130.

Pierson BF (1943) A comparative morphological study of several species of Euplotes closely related to Euplotes patella. Journal of Morphology, 72, 125-165.

Pierson BF, Gierke R, Fisher AL (1968) Clarification of the taxonomic identification of Euplotes eurystomus Kahl and E. aediculatus Pierson. Transactions of the American Microscopical Society, 87, 306-316.

Posada D, Crandall KA (1998) Modeltest: testing the model of DNA substitution. Bioinformatics, 14, 817-818.

Qu ZS, Pan HB, Hu XZ, Li JQ, Al-Farraj SA, Al-Rasheid KAS, Yi ZZ (2015) Morphology and molecular phylogeny of three cyrtophorid ciliates (Protozoa, Ciliophora) from China, including two new species, Chilodonella parauncinata sp. n. and Chlamydonella irregularis sp. n. Journal of Eukaryotic Microbiology, 62, 267-279.

Ronquist F, Huelsenbeck JP (2003) MRBAYES 3: Bayesian phylogenetic inference under mixed models. Bioinformatics, 19, 1572-1574.

Ruffolo JJ (1976) Cortical morphogenesis during the cell division cycle in Euplotes: an integrated study using light optical, scanning electron and transmission electron microscopy. Journal of Morphology, 148, 489-527.

Shao C, Ma HG, Gao S, Khaled ARA, Song WB (2010) Reevaluation of cortical developmental patterns in Euplotes (s. 1.), including a morphogenetic redescription of E. charon (Protozoa, Ciliophora, Euplotida). Chinese Journal of Oceanology and Limnology, 28, 593-602.

Sogin ML, Swanton MT, Gunderson JH, Elwood HJ (1986) Sequence of the small subunit ribosomal RNA gene from the hypotrichous ciliate Euplotes aediculatus. Journal of Protozoology, 33, 26-29.

Song WB, Warren A, Hu XZ (2009) Free-living Ciliates in the Bohai and Yellow Seas, China. Science Press, Beijing. (in Chinese and in English) [宋微波, Warren A., 胡晓钟 (2009) 中国黄渤海的自由生纤毛虫. 科学出版社, 北京.]

Stamatakis A, Hoover P, Rougemont J (2008) A rapid bootstrap algorithm for the RAxML web servers. Systematic Biology, 57, 758-771.

Syberg-Olsen MJ, Irwin NAT, Vannini C, Erra F, Di Giuseppe
G, Boscaro V, Keeling PJ (2016) Biogeography and character evolution of the ciliate genus Euplotes (Spirotrichea, Euplotia), with description of Euplotes curdsi sp. nov. PLoS ONE, 11, e0165442.

Tamura K, Dudley J, Nei M, Kumar S (2007) MEGA4: molecular evolutionary genetics analysis (MEGA) software version 4.0. Molecular Biology and Evolution, 24, 1596-1599.

Tuffrau M (1960) Révision du genre Euplotes, fondée sur la comparaison des structures superficielles. Hydrobiologia, 15, 1-77. (in French)

Vannini C, Ferrantini F, Ristori A, Verni F, Petroni G (2012) Betaproteobacterial symbionts of the ciliate Euplotes: origin and tangled evolutionary path of an obligate microbial association. Environmental Microbiology, 14, 2553-2563.

Washburn ES, Borror AC (1972) Euplotes raikovi Agamaliev, 1966 (Ciliophora, Hypotrichida) from New Hampshire: description and morphogenesis. Journal of Protozoology, 19, 604-608.

Wilbert N (1975) Eine verbesserte Technik der Protargolimprä gnation für Ciliaten. Mikrokosmos, 64, 171-179. (in German)

Xie DM, Fan XP, Ni B, Gu FK (2016) Morphogenesis of the cortical silver-line system in the ciliate genus Euplotes (Protozoa, Ciliophora). Periodical of Ocean University of China (Natural Science), 46, 41-50. (in Chinese with English abstract) [谢冬梅, 范釒金鹏, 倪兵, 顾福康 (2016) 游 仆虫(原生动物, 纤毛门)皮层银线系的形态发生模式. 中 国海洋大学学报(自然科学版), 46, 41-50.]

Yi ZZ, Katz LA, Song WB (2012) Assessing whether alpha-tubulin sequences are suitable for phylogenetic reconstruction of Ciliophora with insights into its evolution in euplotids. PLoS ONE, 7, e40635.

Yi ZZ, Song WB, Clamp JC, Chen ZG, Gao S, Zhang QQ (2009) Reconsideration of systematic relationships within the order Euplotida (Protista, Ciliophora) using new sequences of the gene coding for small-subunit rRNA and testing the use of combined data sets to construct phylogenies of the Diophrys-complex. Molecular Phylogenetics and Evolution, 50, 599-607.

(责任编委: 徐奎栋 责任编辑: 问文杰) 